\title{
Behaviour Profiling in Healthcare Applications Using the Internet of Things Technology
}

\author{
Abdelrahman Abuarqoub
}

\begin{abstract}
This position paper advocates applying the monitoring pogwer of IoT to build profiles of user behaviour using the large volumes of collected data. The desired system exploits sensor data mining approaches to profile user behaviour patterns in smart environments. Sensor data is mined to extract relationships of interest between environmental variables (context) and the user, building in this way behaviour profiles. The capability of applying knowledge to manipulate user's environment is expected take monitoring beyond the simple alert-mode of operation to long term profiling of user's behaviour. After a brief literature review to prove the suitability of IoT as a low-cost unsupervised profiling platform, we give the details of our proposal and the objectives that needs to be met before user behaviour profiling across inter-spaces is possible.
\end{abstract}

Keywords-IoT, behaviour profiling, smart spaces, sensor networks, remote healthcare monitoring.

\section{Introduction}

After the proliferation of processors, wireless connectivity, smartphones and social networking, connecting electronic devices is the next generation of the internet: the Internet of Things (IoT). According to Gartner [1] and ABI research [2], there will be around 26 to 30 billion devices connected to the internet by 2020 . IoT aims at making our daily lives devices and appliances less sophisticated, flexible and highly reachable at any time, from anywhere, to any user across the world. In IoT, smart objects such as home appliances, vehicles, houses, supply chain items, and containers will have digital identities and they will be context aware to sense the environment around them and seamlessly interact with each other. Figure 1 shows a generic scenario of IoT application, where connected things or objects can sense and even influence the real world. The basic notion of the IoT is that objects around us will be connected to the Internet to provide control and contextual services. IoT transforms the everyday physical objects that surround us into an ecosystem of information that will enrich our lives. Smart objects return useful information and use it in various applications during the objects' life cycle.

Abdelrahman Abuarqoub

Faculty of Information Technology

Middle East University

Amman, Jordan

Mohammad Hammoudeh

School of Computing, Mathematics and Digital Technology

Manchester Metropolitan University

Manchester, UK

\author{
Mohammad Hammoudeh
}

IoT offers unprecedented capabilities to face societal challenges by using personalised behaviour change support technologies. It is a promising technology to provide improved healthcare by providing care at home and promoting healthy living through objective feedback.

In recent years, behavioural profiling has emerged in the digital world as a tool to detect and identify criminal and consumer behaviours. It has been mainly used to study how cyber criminals cover their actions to make themselves invisible to security systems. It has also been used in computer tutoring applications, e-commerce, digital marketing, recommender systems, and customers preferences. The Internet of Things (IoT) technology offers a cost-effective platform for monitoring phenomena of interest at high temporal and spatial resolutions. The monitoring power of IoT can be applied to build profiles of user behaviour using the large volumes of collected data.

User behaviour profiling, offers a very beneficial capability to predict humans future actions or detect abnormalities in their behaviour. Behaviour profiling refers to the process of constructing and utilising of users profiles, which are generated using algorithms or mathematical techniques to discover correlations or patterns in large volume of sensor data. The profiling process begins with the description of the applicable problem domain and the specification of the objectives of analysis. Then, data is collected, filtered and analysed in light of the defined domain knowledge. The mined correlations are finally assessed on their validity and relevance by domain experts.

Behaviour profiling technologies can be applied in a variety of different domains, including remote health monitoring. Remote health monitoring is considered one of the most important societal challenges that need to be addressed. Detecting changes and divergence in patient's behaviour can reveal the onset or progression of a disease. Current approaches to user behaviour profiling rely heavily on human intervention and makes limited use of pervasive sensing technologies, such as wearable wireless sensors, to generate behavioural profiles about the monitored patients.

Driven by wider adoption of the calm technology, networks of objects will be increasingly non-invasive, leading to releasing users from the effort of human-to-machine (H2M) interactions and tasks of routine decision-making. Embedded sensor technology is currently more autonomous and pervasive than ever, analysing and deciding for users behind the lines of visibility. The IoT technology is rapidly maturing. Data can now travel transparently and dynamically in and through humans, and over various platforms. The increased availability of smart objects, resulted in heterogeneous Internet that connects spaces to wearable sensors, and even to biosensors inside the human body. 


\section{Libelium Smart World}

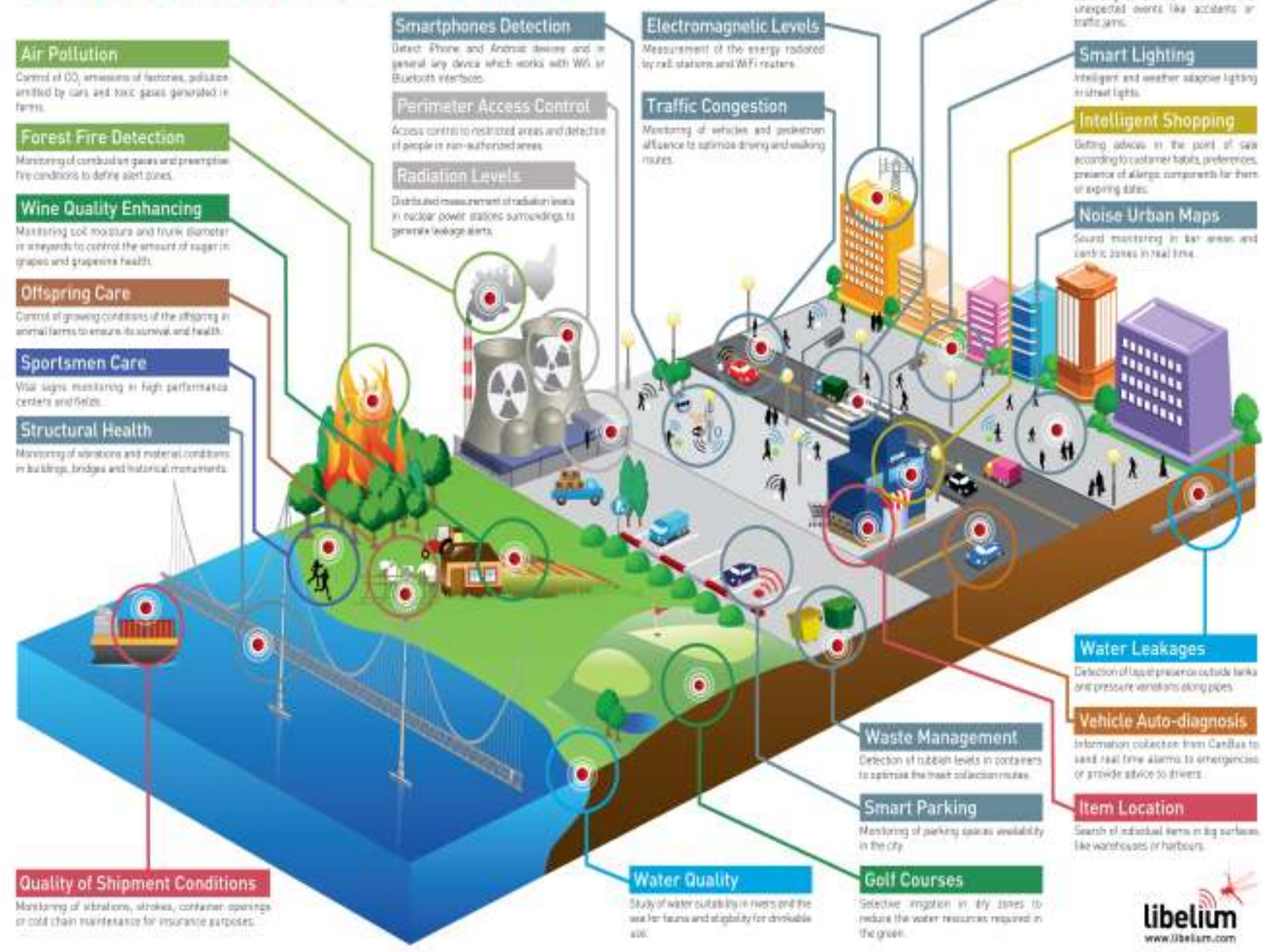

Fig. 1. The IoT generic scenario, adopted from [3]

In this position paper, we attempt to make a case for researchers to investigate how the IoT technology can be used as a platform to profile human behaviour and to develop future connected services. We call for computer, social and psychology scientists and application domain experts to join efforts to model human behaviour to contextualise how, when and where humans interact with the IoT. The ultimate aim is to move the IoT from its current basic instant alertmode of operation to long term monitoring and profiling of user's behaviour using intelligent machine learning and data mining techniques. The long-term user profiling across different administrative spaces allows system designers to develop services that utilises the full potential of the pervasive user monitoring to change the way they behave and experience their lives. This paper probe the importance of ubiquitous technology (i.e., IoT) for behaviour profiling particularly in healthcare applications.

The rest of this paper is organised as follows. Section 2 briefly reviews the current user behaviour profiling techniques. Section 3 presents the idea of using IoT for User profiling in healthcare applications. Section 4 concludes the paper.

\section{Related Work}

Monitoring and profiling of patient's behaviour over an extended period and detecting activity changes is a nontrivial task. Intelligent pattern mining, fusion, and machine learning techniques are needed. Currently, there have been several initiatives to unsupervised user behaviour profiling using wearable and ambient sensing technologies. Some of these prominent and recent approaches are reviewed and discussed in this section.

In earlier studies, such as [4], wearable and blob based ambient sensors were used for observing changes in patients behaviour patterns. To handle continuous activity data streams generated by body sensor networks, a similarity based clustering framework has been proposed. This framework is based on the Hidden Markov Models (HMMs) for clustering behaviour sequences, i.e., user's temporal variation without explicitly defining activities to ensure the privacy of the patient. The proposed framework uses a feature space that is generated using HMMs to express the similarity of sequences to each other. Therefore, the features describing a sequence are calculated as similarity measures between that sequence and other reference sequences that are chosen by specialist from the whole dataset. Detecting 
changes in behaviour patterns is achieved by observing the clustering of sequences in the new feature space. Assuming that certain clusters of behaviour sequences represent normal patient activity over a particular period, outlier can indicate deviations from normal behaviour patterns. If the abnormality is detected, further data analysis can be performed to expose the potential reasons of this deviation.

In [5], the authors presented a pattern mining algorithm for analysing routine behaviour and its deviations. The algorithm obtains a data structure called the routine tree, which is designed to depict user's behaviour patterns. The routine tree is a variable resolution mapping of periods within a day to frequent patterns of activity levels. Each tree represents one day, and each node represents a time interval, for which frequent patterns are stored. The criterion for this period is the continuous activity pattern, where neighbour nodes show a change in the continuous pattern. Better resolution information about a user's behaviour is achieved by moving down the tree and analysing data from nodes nearer the root. The tree is then pruned by merging neighbour time intervals that have the same highest frequency pattern to produce a more compact representation. By combining routine-trees across several days, a graphical view of user behavioural patterns is obtained.

The authors of [5] also proposed a complete and an integrated multi-sensing framework for the development of pervasive systems used in intelligent healthcare delivery and patient monitoring [6]. The framework integrates several hardware and software components and utilises sophisticated data fusion and analysis algorithms, such as [5-7]. Wearable and ambient sensors are used to capture patient activity data.

A similar architecture to [6], called INCASA, is proposed in [8]. INCASA is an integrated solution for profiling patient's behaviour activities. It builds persistent activity records and provides alerts related to significant divergence from normal activity patterns. The architecture consists of a home infrastructure and a remote service provider infrastructure. The former is to collect and store collected data from body and environmental sensors, the latter is to retrieve and analyse personal data as well as updating the activity profiles. Consequently, activity profiles can be presented using a web service interface for the clinical application. INCASA architecture is developed based on the Hydra middleware [9], a project that ease the development of health monitoring architectures. Hydra enables the development of generic services based on open standards. It is capable of routing data, performing session management in the communication phase and synchronising the different entities that are parts of the network in an energy efficient manner.

Another smartphone based behavioural profiling system is proposed in [10]. The authors argue that pervasive healthcare monitoring and behaviour profiling can be often achieved at a lower cost using smartphone applications, rather than using customised wearable sensors. The proposed system builds on the routine mining algorithms proposed in [5]. The authors developed and integrated unsupervised and optimised techniques into a mobile phone application to provide pervasive usability of the system. The application collects data from embedded sensors and clusters it in real-time to estimate the activity of the user.
Next, the data will be conveyed to a server that performs the behaviour profiling using pattern mining techniques.

The above reviewed approaches demonstrates that Wireless Sensor Networks (WSN) and wearable sensors are suitable technologies to provide an effective solution to user monitoring, particularly, in pervasive healthcare applications. However, the ability of these systems to discover new patterns or perform correlations in large volumes of streaming data without necessarily being aggregated in a database is extremely limited. Their other limitations include the difficulty of gathering the required information on the known behaviours and keeping it up to date with new behavioural deviations and environments. They are also application specific and knowledge based systems. Knowledge about behaviours is very specific, dependent on the application domain, hardware platform, available sensing modalities and monitored object characteristics. Consequently, the resulting behaviour profile tool is closely tied to a given environment.

IoT takes the WSN technology to the next level to provide wider contextual services and making it more pervasive. For instance, in healthcare application, medical body sensors can only measure limited vital body conditions, such as skin temperature, pulse rate, sugar level and patient location. While IoT provides more parameters, such as nutrition habits and medicine adherence, which can lead to the discovery of new valuable information with the aid of intelligent mining techniques. Furthermore, IoT relaxes some restrictions and assumptions in the WSN such as, WSN devices are assumed to have limited power, while objects in IoT can be connected to unlimited power source, e.g., smart medicine cabinet, or smart fridge. This allows for in-network filtering and processing of data.

\section{IoT for Behaviour Profiling}

Health and wellbeing is arguably one of the major national and global future societal challenges. Initiatives, such as the Change4Life campaign in the UK [11], aim to deal with the new era of technology-driven attitude and behaviour changes to help people remain active and healthy. The IoT can help to improve our understanding of the causes and mechanisms underlying health and care delivery. To achieve this, we need first to understand how to design, build and study interactive smart spaces that can be adapted to meet the personalised needs of target users.

Currently, we know how to make the technology work, but we do not know whether the intended effects of changed behaviour in relation to achieving, e.g., better health, are always met. One research challenge is to investigate how continuous and understandable visual feedback from the IoT can influence behaviour change in relation to health and wellbeing.

The next generation of IoT should offer the ability to go beyond the simple alert-mode of operation to long term monitoring and profiling of user's behaviour using intelligent machine learning and data mining techniques. To illustrate, medication and lifestyle non-adherence is common in adolescent populations with chronic diseases. Current systems do not go beyond sending a simple alert or reminder for the user to take their medication. In this case, the costs for repeated non-adherence, which can be 
extremely high, are not reported. Beyond that, lifestyle or behaviours often change progressively over a long period of time, e.g., diet and sports. In most cases, people do not realise that their behaviour has changed. It is also not possible to discover such a change by monitoring a single behaviour over a single point of time. Therefore, complex data fusion techniques are needed to learn more about the user and this knowledge can be further used for improving the information retrieval for delivering smarter services to the user. Information about the user can be used to build an ongoing behaviour profile that can be used for analysing user's static and predictable characteristics.

\section{Conclusion}

The proposed research calls for mining IoT data over long periods to go beyond matching predefined behaviours to discover new hidden patterns in the collected data. In current, mainly WSN systems, the extracted patterns are assessed on their validity and relevance by the application domain specialists. The new line of research proposed here aims at maximising information return from IoT data. The focus is to extract behavioural patterns that can only be observed over long time intervals. The observation data is generated by heterogeneous devices, on different platforms and from IoT clouds under different administrative domains. Our brief literature review shows that such a solution is feasible and its benefits are proven.

To address the research questions discussed in Section 3, the research community should prove and test the suitability of the low-cost, autonomous IoT technology for dynamic user behaviour profiling. The testing should cover integrating the WSN with IoT to develop interactive smart spaces that can be adapted to meet the personalised needs of target users. A major research challenge is to allow profiling from multimodal sensors across independent smart spaces or IoT clouds. At the next level, intelligent machine learning and data mining techniques must be investigated for long term monitoring and profiling of users using IoT data. This step may involve the use data fusion to identify progressive long-term changes in users' behaviour. Finally, effective visualisation and reporting methods must be provided to allow users to observe the behavioural patterns they exhibit over a long period.

\section{Acknowledgment}

The authors appreciate the deanship of scientific research at the Middle East University and the Scientific Research Support Fund in Jordan for funding this research.

\section{References}

[1] Gartner. (2016, 12 Jan 2016). Gartner. Available: www.gartner.com/newsroom/id/2636073

[2] ABIresearch. (2013, 7 Jan 2016). More Than 30 Billion Devices Will Wirelessly Connect to the Internet of Everything in 2020. Available: https://www.abiresearch.com/press/more-than-30-billion-devices-willwirelessly-conne/

[3] Libelium_Comunicaciones_Distribuidas_S.L. (2016, 6 Jan 2016). 50 Sensor Applications for a Smarter World. Available: http://www.libelium.com/top_50_iot_sensor_applications_ranking/\#show_i nfographic
[4] L. Atallah, et al., "Behaviour Profiling with Ambient and Wearable Sensing," in 4th International Workshop on Wearable and Implantable Body Sensor Networks (BSN 2007): March 26 - 28, 2007 RWTH Aachen University, Germany, S. Leonhardt, et al., Eds., ed Berlin, Heidelberg: Springer Berlin Heidelberg, 2007, pp. 133-138.

[5] R. Ali, et al., "Pattern mining for routine behaviour discovery in pervasive healthcare environments," in Information Technology and Applications in Biomedicine, 2008. ITAB 2008. International Conference on, 2008, pp. 241-244.

[6] M. ElHelw, et al., "An integrated multi-sensing framework for pervasive healthcare monitoring," in Pervasive Computing Technologies for Healthcare, 2009. PervasiveHealth 2009. 3rd International Conference on, 2009, pp. 1-7.

[7] J. Pansiot, et al., "Ambient and Wearable Sensor Fusion for Activity Recognition in Healthcare Monitoring Systems," in 4th International Workshop on Wearable and Implantable Body Sensor Networks (BSN 2007): March 26 - 28, 2007 RWTH Aachen University, Germany, S. Leonhardt, et al., Eds., ed Berlin, Heidelberg: Springer Berlin Heidelberg, 2007, pp. 208-212.

[8] G. Lamprinakos, et al., "An Integrated Architecture for Remote Healthcare Monitoring," in Informatics (PCI), 2010 14th Panhellenic Conference on, 2010, pp. 12-15.

[9] The_Hydra_project. (2010, 3 Jan 2016). The Hydra project. Available: http://www.hydramiddleware.eu/news.php

[10] R. Ali, et al., "Unsupervised routine profiling in free-living conditions \&\#x2014; Can smartphone apps provide insights?," in Body Sensor Networks (BSN), 2013 IEEE International Conference on, 2013, pp. $1-7$.

[11] Change4Life. (2016, 5 Jan 2016). Change 4 Life. Available: www.change4life.co.uk

About Author (s):

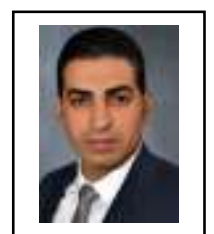

Dr Abdelrahman Abuarqoub is an Assistant Professor in Computer Science in the Faculty of Information Technology at the Middle East University in Jordan. He received his $\mathrm{PhD}$ in Computer Science from the Manchester Metropolitan University in 2014, his MSc (Distinction) in Data Telecommunications and Networks from the University of Salford in 2011, his BSc in Computer Networks Systems from Applied Science University/Jordan in 2009.

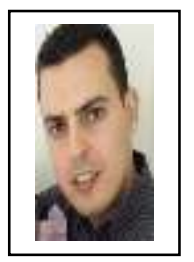

Dr Mohammad Hammoudeh is a Senior Lecturer in Computer Networks and Security in the School of Computing, Math and Digital Technology at the Manchester Metropolitan University. He received his Ph.D. in Computer Science from the University of Wolverhampton in 2009, his MSc (Distinction) in Advanced Distributed Systems from the University of Leicester in 2007, his BSc (Hons) in Computer Communications from Hawaii University in 2004, and a DipCompSci in Computer Information Systems from Siblin Training College in 2002. 